\title{
Blocking TLR2 activity diminishes and stabilizes advanced atherosclerotic lesions in apolipoprotein E-deficient mice
}

\author{
Xiao-xing WANG, Xiao-xi LV, Jia-ping WANG, Hui-min YAN, Zi-yan WANG, Han-zhi LIU, Xiao-ming FU, Zhuo-wei HU* \\ Molecular Immunology and Pharmacology Group, State Key Laboratory of Bioactive Substance and Function of Natural Medicines, \\ Institute of Meteria Medica, Chinese Academy of Medical Sciences \& Peking Union Medical College, Beijing 100050, China
}

\begin{abstract}
Aim: Toll-like receptor 2 (TLR2) signaling plays a critical role in the initiation of atherosclerosis. The aim of this study was to investigate whether blocking TLR2 activity could produce therapeutic effects on advanced atherosclerosis.

Methods: Forty-week old apolipoprotein E-deficient $\left(A p o E^{-/-}\right)$mice fed on a normal diet were intravenously injected with a TLR2-neutralizing antibody or with an isotype-matched IgG for 18 weeks. Double-knockout ApoE ${ }^{-/-}$TIr2 $^{-/-}$mice were taken as a positive control. At the end of the treatments, the plasma lipid levels were measured, and the plaque morphology, pro-inflammatory cytokines

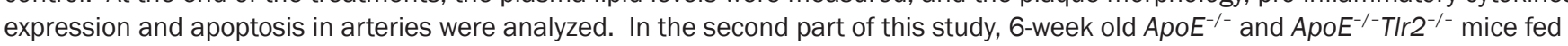
on a high-cholesterol diet for 12 to 24 weeks, the expression levels of TLR2 and apoptotic markers in arteries were examined.

Results: Blockade of TLR2 activity with TLR2-neutralizing antibody or knockout of TIr2 gene did not alter the plasma lipid levels in $A p o E^{-/-}$mice. However, the pharmacologic and genetic manipulations significantly reduced the plaque size and vessel stenosis, and increased plaque stability in the brachiocephalic arteries. The protective effects of TLR2 antagonism were associated with the suppressed expression of pro-inflammatory cytokines IL- 6 and TNF- $\alpha$ and the inactivation of transcription factors NF-KB and Stat3. In addition, blocking TLR2 activity attenuated ER stress-induced macrophage apoptosis in the brachiocephalic arteries, which could promote the resolution of necrotic cores in advanced atherosclerosis. Moreover, high-cholesterol diet more prominently accelerated atherosclerotic formation and increased the expression of pro-apoptotic protein $\mathrm{CHOP}_{\text {and }}$ apoptosis in $\mathrm{ApoE}^{-/-} \mathrm{mice}$ than in $\mathrm{ApoE}^{-/-}$ TIr2 ${ }^{-/-}$mice.

Conclusion: The pharmacologic or genetic blockade of TLR2 activity diminishes and stabilizes advanced atherosclerotic lesions in $\mathrm{ApoE}^{-/-}$mice. Thus, targeting TLR2 signaling may be a promising therapeutic strategy against advanced atherosclerosis.
\end{abstract}

Keywords: toll-like receptor 2; cardiovascular disease; atherosclerosis; inflammation; macrophage; ER stress; apoptosis; apolipoprotein E-deficient $\left(A p o E^{-/-}\right)$mice

Acta Pharmacologica Sinica (2013) 34: 1025-1035; doi: 10.1038/aps.2013.75; published online 15 Jul 2013

\begin{abstract}
Introduction
Atherosclerosis is a common cause of cardiovascular disease. Cardiovascular disease is the leading cause of death and poses an increasing threat to human health worldwide ${ }^{[1]}$. Epidemiological and experimental studies have established a predominant role for inflammation in all stages of atherosclerosis, from the formation of early stable atherosclerotic plaques to advanced plaques that are prone to rupture ${ }^{[2-5]}$. Recently, several studies have focused on the effects of pharmacological interventions on the stability of advanced plaques because the rupture of unstable lesions contributes to acute coronary syn-
\end{abstract}

\footnotetext{
* To whom correspondence should be addressed.

E-mail huzhuowei@imm.ac.cn

Received 2013-03-07 Accepted 2013-05-09
}

drome ${ }^{[4,6,7]}$. Unstable plaques are characterized by large, lipidfilled necrotic cores encapsulated by a thin fibrous cap, high levels of pro-inflammatory mediators and matrix proteases, and apoptosis. The development of unstable plaques is associated with the involvement of both the innate and adaptive immune systems.

Toll-like receptors (TLRs) are a family of pattern-recognition receptors that play a key role in innate immunity and initiate inflammatory responses. The ligation of these receptors initiates the activation of nuclear factor- $\mathrm{KB}(\mathrm{NF}-\mathrm{\kappa B})$, resulting in the expression of a wide array of inflammatory genes ${ }^{[8,9]}$. Among the characterized TLRs, TLR2 is unique by virtue of its ability to heterodimerize with TLR1 or TLR6, resulting in relatively broad ligand specificity, including both endogenous and exogenous ligands ${ }^{[10,11]}$. It is noteworthy that all of the 
potential endogenous TLR2 agonists, including biglycan, high-mobility box chromosomal protein 1 (HMGB1) and components of oxidized lipoproteins, are found in atherosclerotic lesions ${ }^{[12-14]}$. In addition, the TLR2 signaling pathway is associated with the endoplasmic reticulum (ER) stress response. ER stress can increase TLR2 expression in vitro and in vivo, and TLR2 contributes to apoptosis in macrophages undergoing lipid-triggered ER stress ${ }^{[15]}$. Thus, TLR2 may be a potential target for therapeutic interventions in the treatment of atherosclerosis.

Indeed, TLR2 ligands and increased TLR2 expression have been observed in human atherosclerotic plaques, and the important role of TLR2 in inflammation and matrix degradation is indicated by the cells found in human carotid atherosclerotic lesions ${ }^{[16,17]}$. Moreover, TLR2 gene deficiency in a mouse model significantly inhibited the progression of early atherosclerosis $^{[18,19]}$. However, there are limited data regarding the effects of TLR2 on advanced lesions in animal models. Using pharmacological and genetic inhibition of TLR2 activity, we investigated the therapeutic and prophylactic effects of TLR2 blockade on advanced atherosclerotic plaques in the brachiocephalic artery of chow-fed apolipoprotein E (ApoE)deficient mice, which have never been evaluated before. We found that blocking TLR2 activity reduces and stabilizes advanced atherosclerotic lesions in ApoE-deficient mice via the attenuation of inflammation and ER-stress in blood vessels. Our work indicates that TLR2 activity plays a crucial role in the pathogenesis of advanced atherosclerosis and that blocking this signaling pathway may be a promising strategy for the treatment of atherosclerosis and its complications.

\section{Materials and methods}

\section{Animals and experimental design}

$\mathrm{ApoE^{-/- }} \mathrm{Tlr}^{-2^{-1}}$ mice were generated from heterozygous intercrosses of $\mathrm{ApoE}^{-/-}$mice (C57BL/ 6 background, Jackson Laboratory) and $\mathrm{Tlr}^{-/-}$mice (C57BL/ 6 background, Jackson Laboratory). The genotypes of the homozygous double-knockout or ApoE single-knockout mice were confirmed using PCR, as previously described ${ }^{[18]}$. All animals were housed under standard conditions for humidity, room temperature, and darklight cycles, with access to water and food ad libitum. The studies were conducted according to the principles outlined by the Institutional Committee for the Ethics of Animal Care and Treatment in Biomedical Research (Beijing, China).

For the first experiment, all of the mice were maintained on a normal chow diet [4\% (wt/wt) fat, $20 \%(\mathrm{wt} / \mathrm{wt})$ protein, GB-14924]. At 40 weeks of age, the $\mathrm{ApoE}^{-/-} \mathrm{Tlr}^{+/+}$mice were randomly divided into 3 groups ( $n=16$ per group) and treated with saline, an isotype-matched IgG antibody (SouthernBiotech, Birmingham, AR, USA), or a TLR2-neutralizing antibody (R\&D System, Minneapolis, MN, USA) intravenously. The dosing and timing of antibody (ab) administration were determined based on our previous studies ${ }^{[20,21]}$. The first dose treatment regimen was $200 \mu \mathrm{g} / \mathrm{kg}$ every $4 \mathrm{~d}$ for 2 weeks, and the remaining doses were $100 \mu \mathrm{g} / \mathrm{kg}$ given once per week for an additional 16 weeks. The $A p o E^{-/-} \mathrm{Tlr}^{-/-}$mice, termed the double-knockout (DK) group, were kept under the same conditions. All of the mice were sacrificed at 58 weeks of age. In the second experiment, the $\mathrm{ApoE}^{-/-} \mathrm{Tlr} 2^{+/+}$and $\mathrm{ApoE^{-/- }} \mathrm{Tlr}^{-2^{-/}}$ mice were fed an atherogenic high-cholesterol (HC) diet [15.8\% (wt/wt) fat, 1.25\% (wt/wt) cholesterol] from 6 weeks of age and were sacrificed for analysis at 12, 18, and 24 weeks of age ( $n=8$ per group).

\section{Tissue collection and processing}

After the mice had fasted for $4 \mathrm{~h}$, blood was obtained via the retro-orbital venous plexus for lipid measurements. The mice were then anesthetized using $50 \mathrm{mg} / \mathrm{kg}$ ip pentobarbital and perfused through the left cardiac ventricle with ice-cold phosphate-buffered saline under physiological pressure. The aortas were excised quickly, snap frozen from the aortic arch to the iliac bifurcation, and maintained at $-80^{\circ} \mathrm{C}$ prior to protein analysis. The entire brachiocephalic artery from each animal was fixed in $4 \%$ paraformaldehyde for $24 \mathrm{~h}$ at $4{ }^{\circ} \mathrm{C}$, embedded in paraffin, and serially sectioned ( $5 \mu \mathrm{m}$ thickness).

\section{Biochemical analysis}

The levels of total cholesterol, high-density lipoprotein cholesterol (HDL-C), low-density lipoprotein cholesterol (LDL-C) and triglycerides in the plasma were determined using commercial kits (Biosino, Beijing, China) according to the manufacturers' instructions.

\section{Histological and immunohistochemical staining}

Every fifth section of the serial sections was stained with a modified Movat's pentachrome stain or Sirius red to evaluate the composition and stability of advanced atherosclerotic plaques in the brachiocephalic artery, and 12-15 sections per mouse were analyzed for each stain. The stained sections were photographed under 200×magnification, and 2-3 fields of view per section that contained the full lesional area were captured and analyzed using Image-Pro Plus 5.0 (Media Cybernetics, Bethesda, MD, USA). The average of all sections represented the atherosclerotic lesion of one mouse.

For immunohistochemical staining, at least 4 sections of the brachiocephalic artery per mouse were incubated with a rat anti-mouse macrophage antibody (Mac-3, 1:50, BD, Franklin Lakes, NJ, USA), a mouse monoclonal antibody to smooth muscle actin (a-SMA, 1:100, Santa Cruz Biotechnology, CA, USA), or a rabbit anti-matrix metalloproteinase (MMP)-2 antibody (1:100, Abcam, Cambridge, UK). The bound antibodies were complexed with peroxidase-linked secondary antibodies and detected using diaminobenzidine. The stained sections were photographed under $200 \times$ magnification, and 2-3 fields of view that contained the full lesional area per section were captured and analyzed using Image-Pro Plus 5.0. The average of all sections represented the expression of target proteins in the atherosclerotic lesion.

To assess the co-localization of macrophages and C/EBP homologous protein $(\mathrm{CHOP})$, a rat anti-mouse macrophage antibody (Mac-3, 1:50, BD, Franklin Lakes, NJ, USA) and a mouse monoclonal antibody to CHOP (1:100, Cell Signaling 
Technology, Danvers, MA, USA) were used as the primary antibodies. Four tissue sections per mouse were incubated with Alexa 647-labeled chicken anti-rat and Alexa 488-labeled rabbit anti-mouse secondary antibodies (1:200, Invitrogen, Carlsbad, CA, USA). The sections were imaged using an E2000U confocal microscope and evaluated using Leica TCS SP2 software.

\section{In situ TdT-mediated dUTP nick-end labeling assays}

Apoptotic cells in atherosclerotic lesions were detected with the TdT-mediated dUTP nick-end labeling (TUNEL) technique using kits (Promega, Madison, WI, USA). The nuclei were stained with DAPI. TUNEL-positive nuclei were counted under an Olympus I720 inverted fluorescent microscope. To assess macrophage co-localization, the macrophages were detected using a rat anti-mouse macrophage antibody (Mac-3, 1:50, BD, Franklin Lakes, NJ, USA) followed by an Alexa 647-labeled chicken anti-rat secondary antibody (1:200, Invitrogen, Carlsbad, CA, USA).

\section{Western blots}

After tissue homogenization, the extracted proteins were separated using $12 \%$ SDS-PAGE and subjected to immunoblotting with specific antibodies, including anti-MMP-2 (Abcam, Cambridge, UK), anti-phospho-NF-кB p65, anti-NF-кB p65, anti-cleaved caspase-3, anti-CHOP (Cell Signaling Technology, Danvers, MA, USA), anti-phospho-Stat3, anti-Stat3, antiinterleukin (IL)-6, anti-IL-10 or anti-tumor necrosis factor (TNF)- $a$ (Santa Cruz Biotechnology, CA, USA). The secondary antibody signals were detected using ECL Plus Western blot reagents (Amersham Biosciences, PA, USA) according to the manufacturer's instructions.

\section{Statistical analysis}

The data are presented as the mean \pm SEM. The comparisons among several groups were performed with a one-way ANOVA using SPSS. Data that did not demonstrate a normal distribution were analyzed using a Mann-Whitney U test. $P<0.05$ was considered to be significant.

\section{Results}

Blocking TLR2 activity reduces and stabilizes atherosclerotic lesions in the brachiocephalic artery

At the time of sacrifice, mice that had been treated with a TLR2-neutralizing antibody and TLR2-deficient mice displayed similar plasma levels of total cholesterol, LDL-C, HDL-C, and triglycerides as their ApoE-deficient littermates (Figure 1), indicating that TLR2 blockade did not significantly alter the blood lipid profile. Movat's staining was used to evaluate plaque composition during brachiocephalic atherosclerosis in each group (Figures 2A and 2B). As expected, blocking TLR2 activity through either TLR2ab administration for 18 weeks or TLR2 gene depletion significantly decreased atherosclerotic lesion formation, as evidenced by the reductions in the mean lesion area, and maximum lesion stenosis, compared to mice that had been treated with IgG or saline
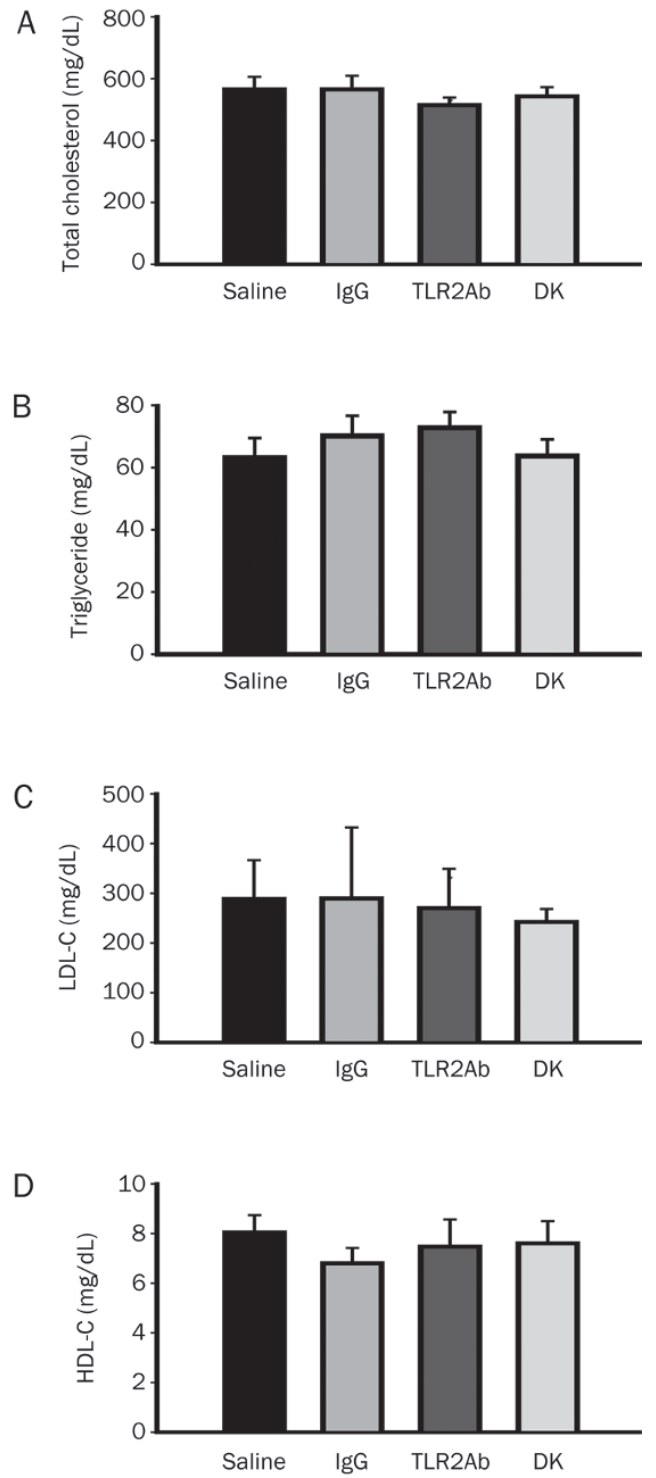

Figure 1. Blocking TLR2 activity does not affect plasma lipid levels. The total cholesterol $(A)$, triglyceride $(B), L D L-C(C)$, and HDL-C (D) levels were determined in 58-week-old $A p o E^{-/-}$mice treated with saline, IgG or a TLR2ab iv for 18 weeks and in TLR2-deficient $A p o E^{-/-}$mice (DK) fed a chow diet. The data are shown as the mean $\pm \operatorname{SEM}(n=12$ mice/group).

$(P<0.05$, Figures $2 \mathrm{C}$ and $2 \mathrm{D})$. In addition, significant reductions in the size of necrotic cores (normalized to lesion area) and increases in media integrity were observed in the TLR2ab-treated and TLR2-deficient groups compared to the IgG- and saline-treated groups ( $P<0.05$, Figures $2 \mathrm{E}$ and $2 \mathrm{~F}$ ), suggesting that the blockade of TLR2 activity promotes plaque stability in advanced atherosclerotic lesions in ApoE-deficient mice. This finding was further supported by the histological analysis of collagen and a-SMA, which may protect atherosclerotic plaques from rupture, as well as macrophage accumulation, which contributes to plaque rupture in brachiocephalic lesions. The collagen content and a-SMA expression were both significantly increased, and the accumulation of mac- 
A

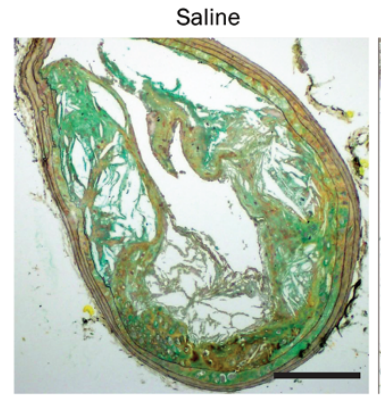

B

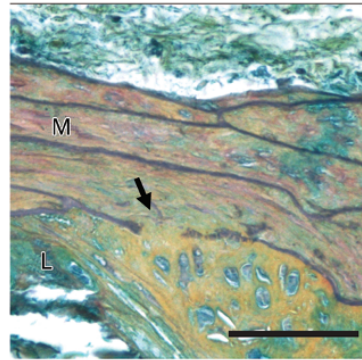

IgG
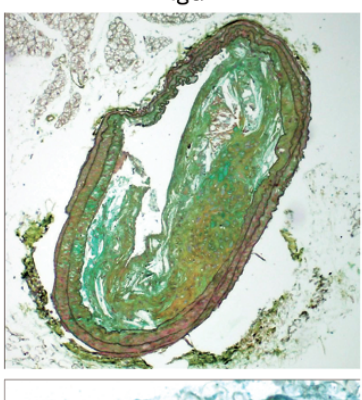
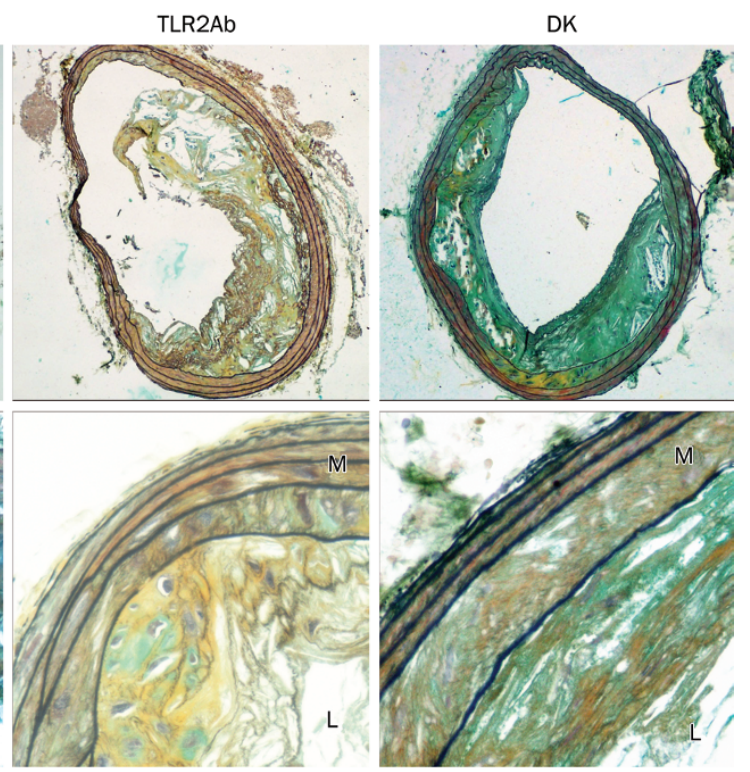

C

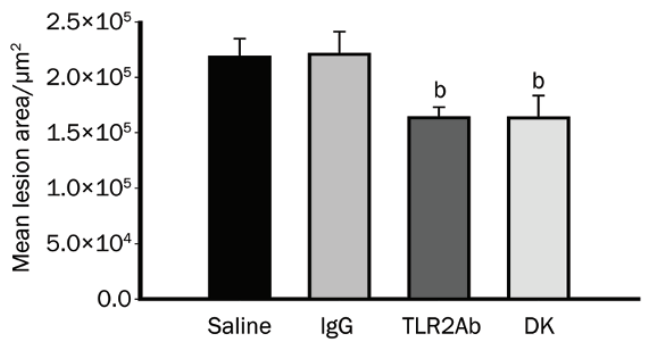

D

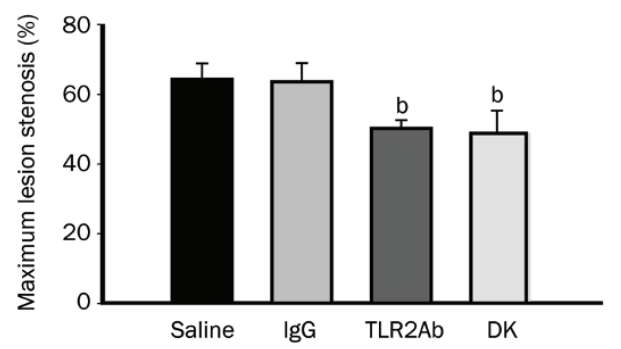

E

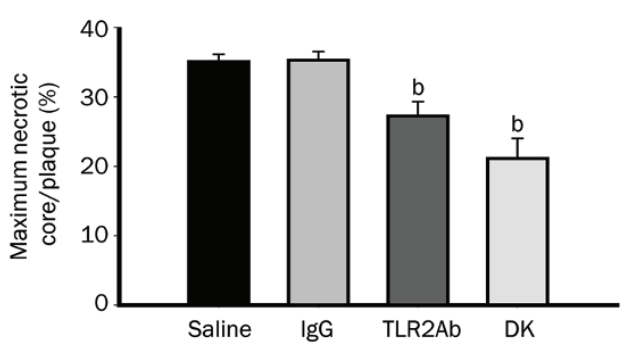

F

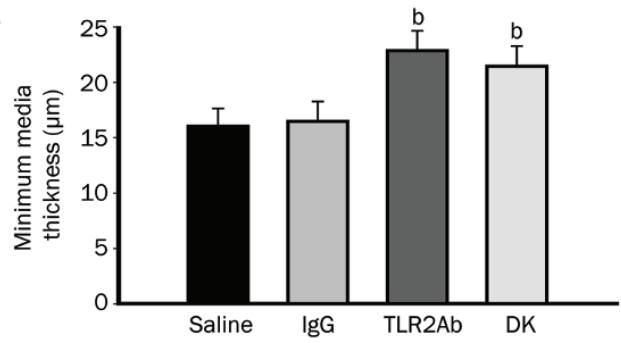

Figure 2. TLR2 blockade reduces and stabilizes advanced atherosclerotic plaques in the brachiocephalic artery. (A and B) Movat's staining showed the composition of advanced lesions from saline-, IgG-, and TLR2ab-treated and TLR2-deficient groups. The arrows indicate erosion of the media. The scale bars represents $200 \mu \mathrm{m}$ in Panel A and $50 \mu \mathrm{m}$ in Panel B. TLR2ab administration for 18 weeks and TLR2 deficiency significantly reduced the mean lesion area (C), maximum lesion stenosis (D), maximum necrotic cores/plaque (E), and minimum media thickness (F) in ApoE-deficient mice. L, lumen; M, media. The data are shown as the mean \pm SEM ( $n=10$ mice/group). ${ }^{b} P<0.05$ vs the saline-treated group.

rophages was significantly suppressed in TLR2ab-treated and TLR2-deficient mice compared with the other groups $(P<0.05$, Figures 3).

\section{TLR2 blockade attenuates inflammation in atherosclerotic} arteries

Because no significant differences in the lipid levels were observed, the therapeutic efficacy of targeting TLR2 may depend on the alteration of the inflammatory response. In addition to the diminished accumulation of macrophages, the expression of MMP-2, one of the most important MMPs, was significantly suppressed in the lesions of ApoE-deficient mice treated with the TLR2ab and those lacking the TLR2 gene, as demonstrated by both the immunohistochemical straining and immunodetection of aorta protein extracts $(P<0.05$, Figures $4 \mathrm{~A}$ and $4 \mathrm{~B})$. Although the expression of the pro-inflammatory transcription factor NF-kB p65 subunit was not significantly altered, its phosphorylation level was significantly decreased by TLR2 inhibition, as compared to mice treated with IgG or saline $(P<0.05$, Figure $4 C)$. To further analyze the effects of 

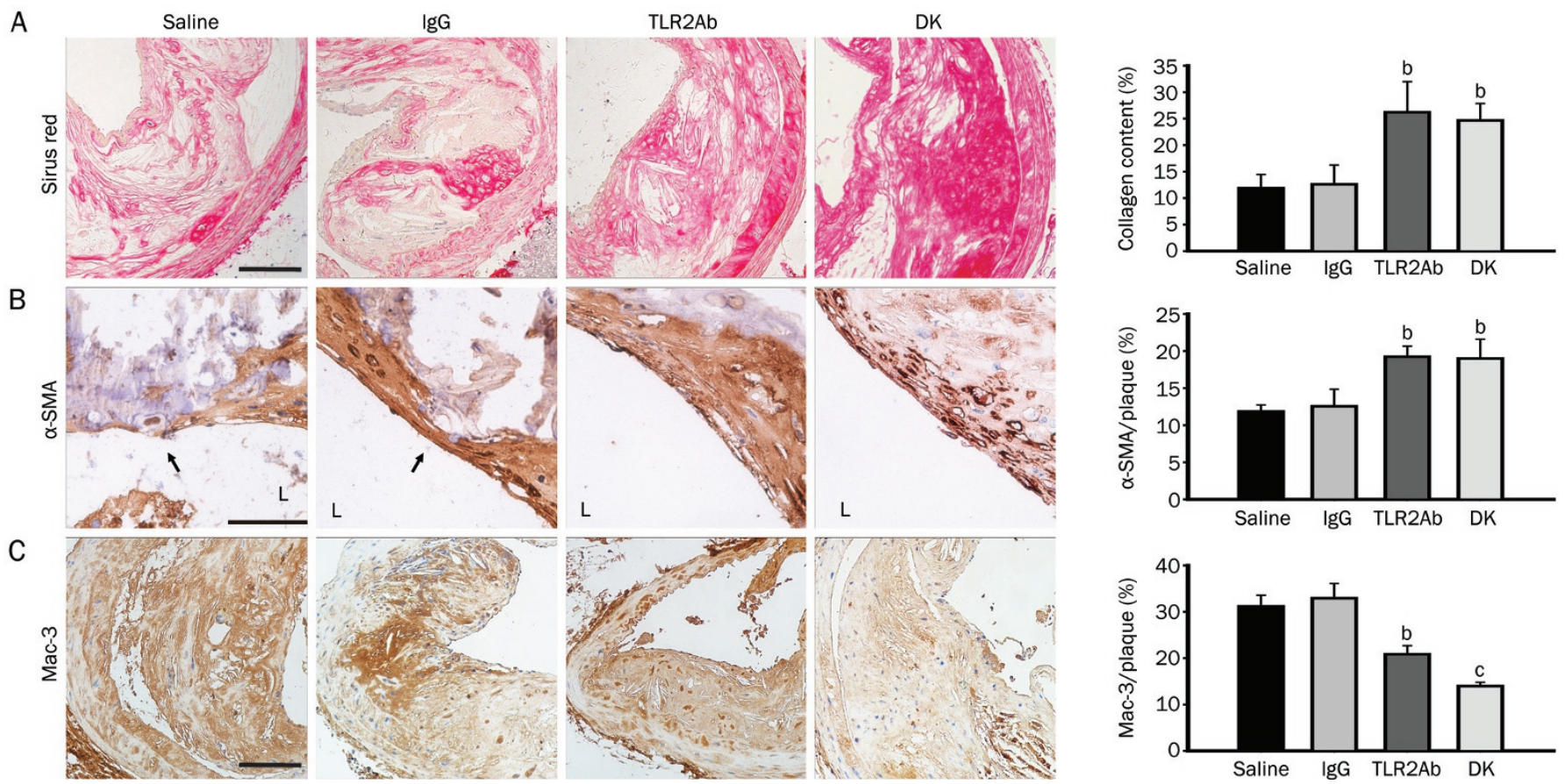

Figure 3. TLR2 blockade stabilizes advanced atherosclerotic plaques. In the TLR2ab-treated and TLR2-deficient groups, Sirius red staining revealed increased collagen content (A). Immunohistochemical staining revealed more abundant smooth muscle cell distribution (B) and decreased macrophage accumulation $(C)$ in lesions in the brachiocephalic artery. The scale bars represent $50 \mu \mathrm{m}$. L indicates the lumen. The data are shown as the mean \pm SEM ( $n=10$ mice/group). ${ }^{b} P<0.05,{ }^{c} P<0.01$ vs the saline-treated group.

TLR2 blockade on inflammation in atherosclerosis, the levels of the pro-inflammatory cytokines IL- 6 and TNF- $\alpha$ were measured in aorta protein extracts from the different groups. Blocking TLR2 activity significantly reduced IL- 6 and TNF- $a$ expression, compared with IgG or saline treatment $(P<0.05$, Figure $4 \mathrm{E}$ ). Interestingly, we found that the expression or phosphorylation of the immunosuppressive cytokine IL-10 and transcription factor Stat3 (signal transducer and activator of transcription-3) were significantly inhibited in the TLR2abtreated and TLR2-deficient groups, as compared to the IgG- or saline-treated groups $(P<0.05$, Figure $4 \mathrm{D}$ and $4 \mathrm{E})$. This finding indicates that the therapeutic effect of blocking TLR2 activity on atherosclerotic lesions is also associated with an attenuation of the immunosuppressive environment, in which Stat3 and IL-10 play critical roles.

\section{TLR2 blockade decreases macrophage apoptosis and CHOP} expression in advanced atherosclerotic lesions

In advanced atherosclerotic lesions, macrophage apoptosis is associated with the development of plaque necrosis, a key feature of plaque rupture. Therefore, TUNEL analysis was used to assess the effect of TLR2 blockade on lesional macrophage apoptosis. The quantification of the data, represented as the percentage of apoptotic cells, revealed an approximately $64.4 \%$ decrease in the TLR2ab-treated group and an approximately $59.6 \%$ decrease in the TLR2-deficient group compared with the IgG- or saline-treated groups ( $P<0.05$, Figures $5 \mathrm{~A}$ and $5 \mathrm{~B})$. Cellular immunostaining revealed that many of the TUNEL- positive nuclei were located in macrophage-rich regions (Figure 5A). Similar results were obtained from the aorta protein extracts using activated caspase- 3 as a marker for apoptosis (Figure 5C). To determine whether the anti-apoptotic effects of blocking TLR2 activity were associated with CHOP signaling, CHOP expression was analyzed at the protein level. A large inhibitory effect was observed in mice treated with the TLR2ab and in TLR2-deficient mice, compared to mice that had been treated with IgG or saline $(P<0.01$, Figure 5D). Double immunostaining for $\mathrm{CHOP}$ and Mac-3 in plaques revealed partial co-localization of both proteins, suggesting that CHOP was induced in macrophages that invaded atherosclerotic lesions. In addition, TLR2 inhibition resulted in visibly weaker co-localization of CHOP and macrophages (Figure 5E).

TLR2 mediates accelerated atherosclerotic formation and regulates $\mathrm{CHOP}$ expression and apoptosis in ApoE-deficient mice fed $\mathrm{HC}$ diets

Six-week-old ApoE $E^{-/-} \mathrm{Tlr}^{+/+}$and $A p o E^{-/-} \mathrm{Tlr}^{-/-}$mice were fed an atherogenic $\mathrm{HC}$ diet to accelerate lesion formation. Consistent with previous studies, after 18 weeks on the atherogenic diet, TLR2 deficiency resulted in decreased mean lesion areas and necrotic core sizes, as indicated by Movat's staining (Figures $6 \mathrm{~A}-6 \mathrm{C}$ ). Along with the development of atherosclerosis, TLR2 and macrophage co-localization in plaques was observed in $A p o E^{-/-} \mathrm{Tlr}^{+/+}$mice via double immunofluorescence staining (Figure 6D). To provide further evidence of the relevance of TLR2 and macrophages in apoptosis during atherosclerosis, 
A

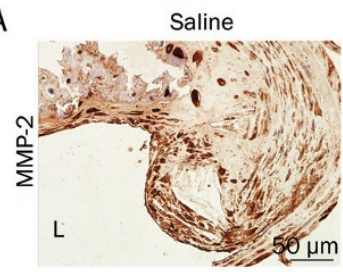

B
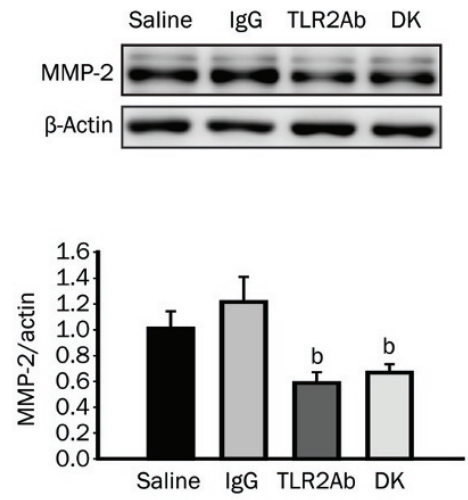

IgG

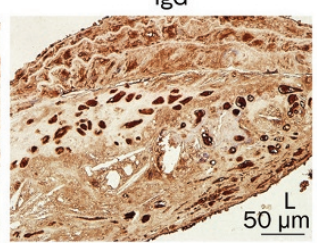

TLR2Ab
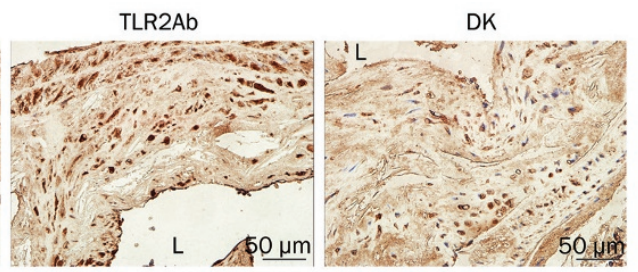

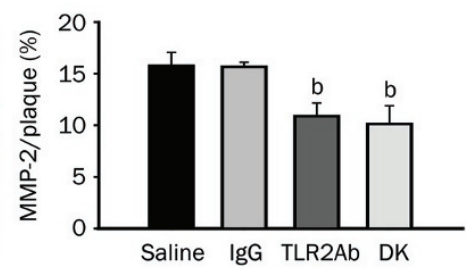

C
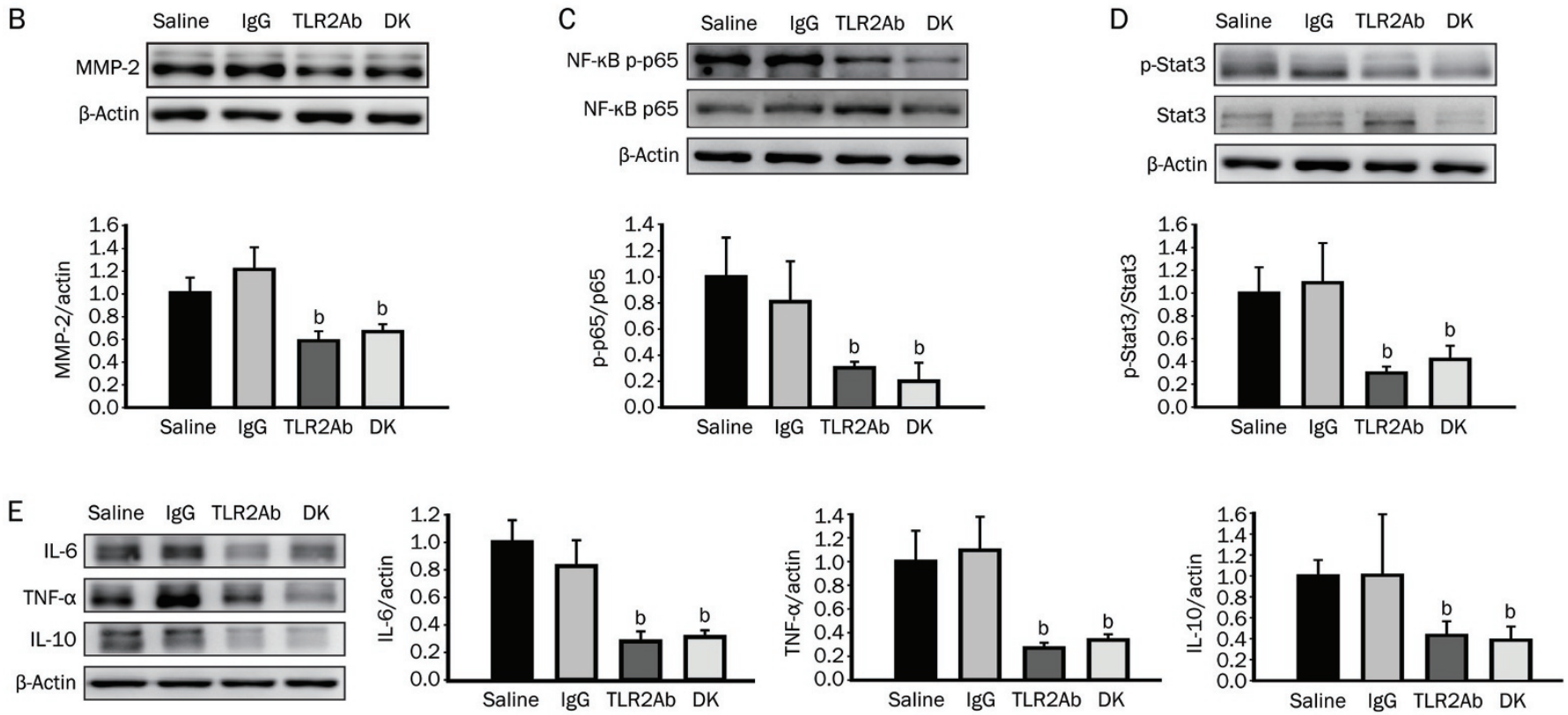

Figure 4. TLR2 blockade inhibits vascular inflammation in atherosclerotic arteries. MMP-2 expression (A and B), NF-KB p65 phosphorylation (C), Stat3 phosphorylation (D) and the expression of the cytokines IL-6, TNF- $\alpha$, and IL-10 (E) were reduced in the TLR2ab-treated and TLR2-deficient groups. The scale bars represent $50 \mu \mathrm{m}$. The data are shown as the mean \pm SEM ( $n=6$ mice/group). ${ }^{b} P<0.05$ vs the saline-treated group.

the expression of activated caspase-3, the proapoptotic protein $\mathrm{CHOP}$ and its main downstream signaling molecule Bcl-2 were detected. The greater levels of activated caspase- 3 indicated that apoptosis had increased, and $\mathrm{CHOP}$ expression was also significantly elevated in the aortas of $\mathrm{ApoE}^{-/-} \mathrm{Tlr}^{+/+}$mice after the $\mathrm{HC}$ diet was introduced. In addition to its protective effects against lesion formation, TLR2 deficiency decreased the expression of activated caspase- 3 and $\mathrm{CHOP}$ and enhanced the expression of the survival signal Bcl-2, compared with Apo $E^{-/-} \mathrm{Tlr}^{+/+}$mice, especially at 24 weeks of age $(P<0.05$, Figures $6 \mathrm{E}-6 \mathrm{G})$.

\section{Discussion}

Growing evidence of the involvement of TLR2 activation in atherogenesis has been obtained using mouse models. The targeted deletion of TLR2 in ApoE $\mathrm{E}^{-/-}$or $\mathrm{Ldlr}^{-/-}$mice resulted in a significant reduction in the number of atherosclerotic lesions within aortas, as assessed by en face or aortic sinus analysis, in mice on an HC diet or a normal chow diet ${ }^{[18,19]}$. In addition, the administration of TLR2 ligands, including synthetic compounds and live bacteria, significantly aggravated atherosclerosis ${ }^{[22,23]}$. However, these studies were only concerned with the pathogenesis of early atherosclerosis, and there are no reports about the effects of TLR2 on plaque stability during advanced atherosclerosis. Chow-fed older ApoEdeficient mice that develop advanced atherosclerotic lesions in the brachiocephalic artery were recently reported to share many of the morphologic features of advanced disease in humans ${ }^{[24-26]}$. Compared with lesions within the aortas and aortic sinus, which are analogous to early fibrous cap atheromas in humans, plaques in the brachiocephalic arteries exhibit more features of advanced disease, such as necrotic cores, media erosion and the loss of fibrous cap continuity ${ }^{[7]}$. Using this animal model, we demonstrated that TLR2 deficiency reduces and stabilizes advanced atherosclerotic lesions in the brachiocephalic artery of older ApoE-deficient mice by decreasing the plaque size and the number of necrotic cores, maintaining media integrity and elevating the expression of collagen and a-SMA. Importantly, we found that the therapeutic inhibition of TLR2 activity by a TLR2-neutralizing antibody produced anti-atherosclerotic effects on established atherosclerosis in ApoE-deficient mice and that these effects were identical to those induced by TLR2 gene depletion. Our work may pave the way for a pharmacological intervention of advanced atherosclerosis.

Inflammatory processes play a pivotal role in the devel- 
A
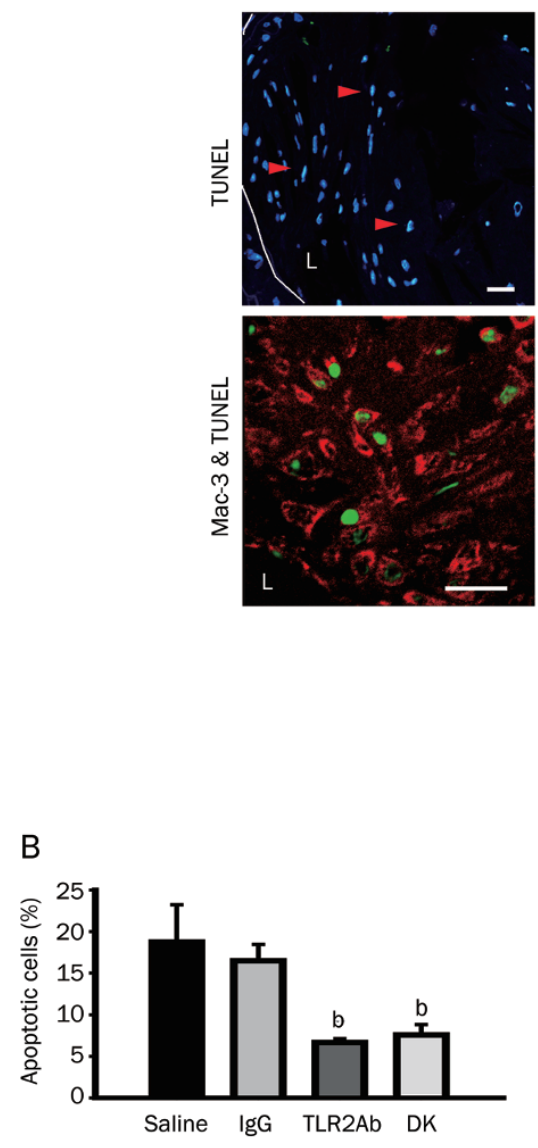
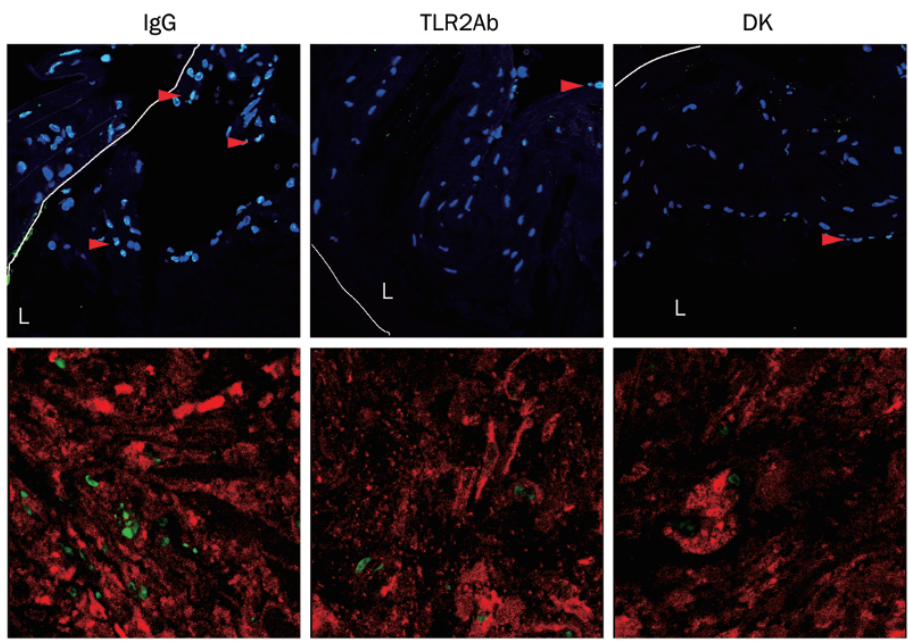

C
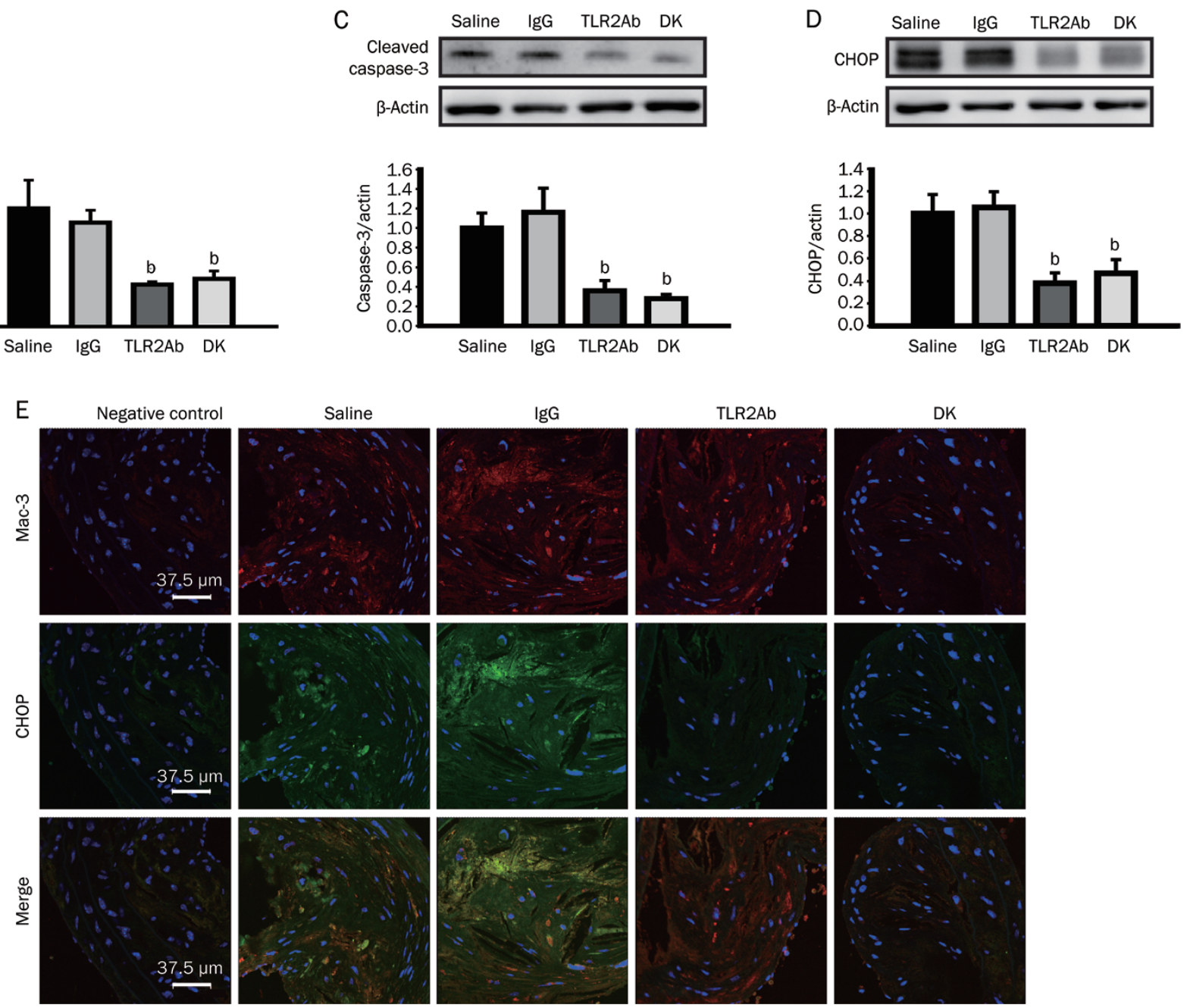

Figure 5. TLR2 blockade inhibits macrophage apoptosis and CHOP expression in atherosclerotic lesions in the brachiocephalic artery. (A) TUNEL analysis showed reduced macrophage apoptosis in the plaques of $A p o E^{-/-}$mice treated with the TLR2ab and in ApoE $E^{-/-} T_{1 / 22^{-/-}}$mice. The statistical analysis is shown in (B). Cleaved caspase-3 (C) and CHOP (D) expression in the aortas and the co-localization of macrophages (Mac-3) and $\mathrm{CHOP}(\mathrm{E})$ in advanced plaques were evaluated for the four sample groups. The scale bars represent $37.5 \mu \mathrm{m}$. The data are shown as the mean \pm SEM $(n=6$ mice/ group). ${ }^{\mathrm{b}} \mathrm{P}<0.05$ vs the saline-treated group. 
A

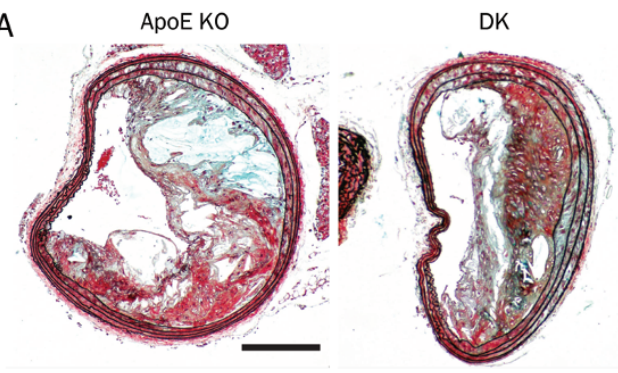

B

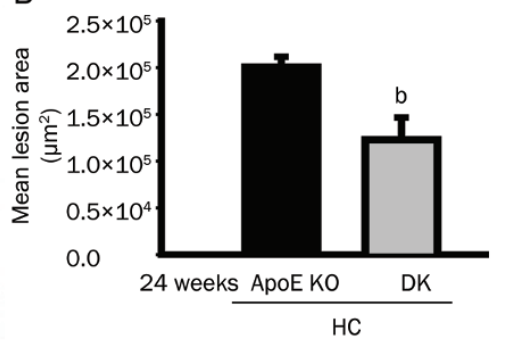

C

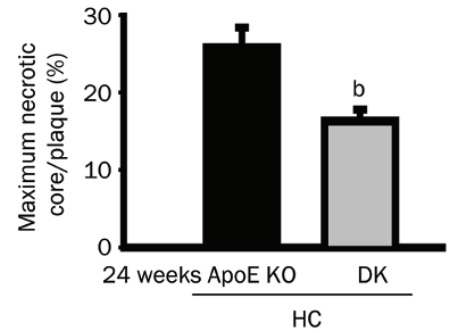

D
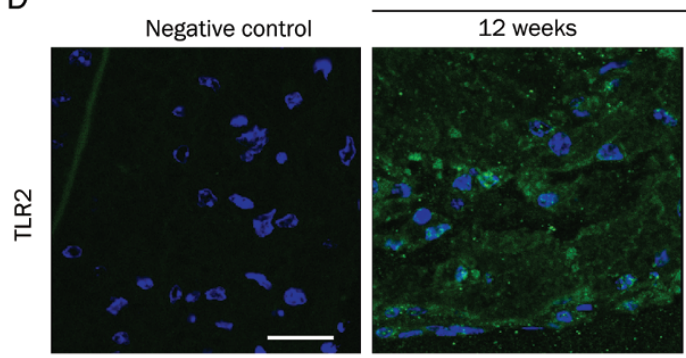

ApoE KO
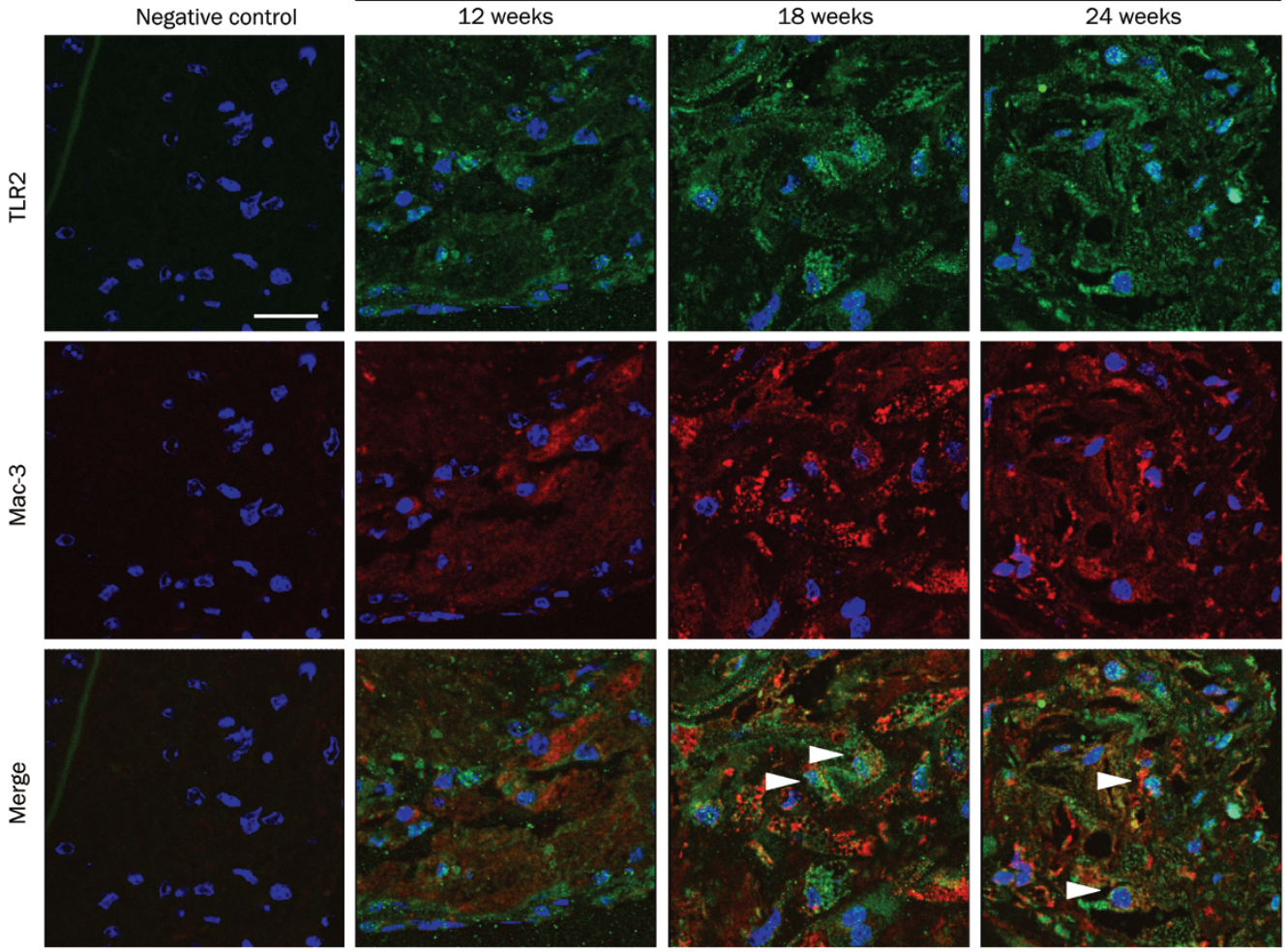

E

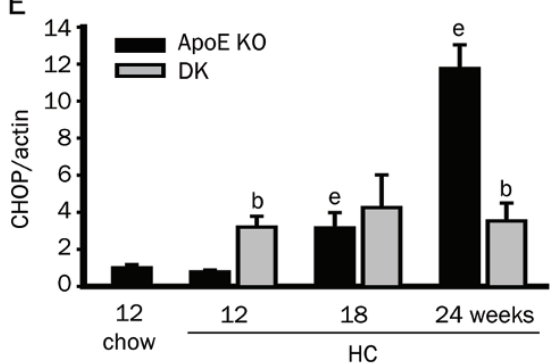

$\mathrm{F}$

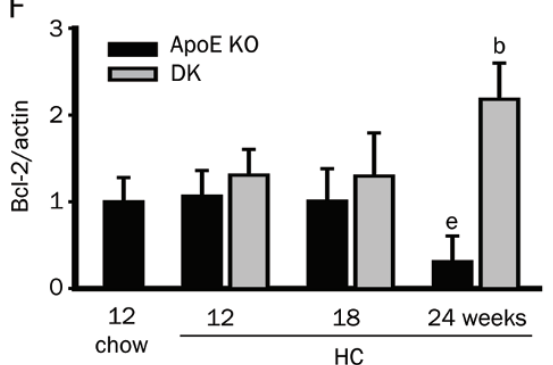

G

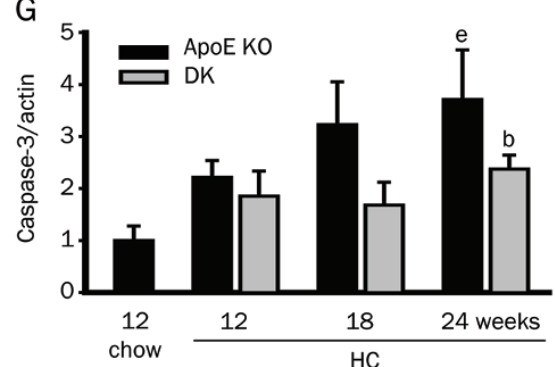

Figure 6. High cholesterol $(\mathrm{HC})$ diets increase the expression of TLR2 in macrophages and blockade of TLR2 decreases the HC-induced expression of $\mathrm{CHOP}$ and apoptosis in atherosclerotic lesions. (A) Movat's staining revealed plaque morphology in the brachiocephalic arteries of $A p o E^{-/-}$T/r2 $2^{+/+}$ and $\mathrm{ApoE}^{-1-} \mathrm{Tlr}^{-/-}$mice. The scale bars represent $200 \mu \mathrm{m}$. TLR2 deficiency significantly reduced the mean lesion areas (B) and the sizes of the necrotic cores (C) ( $n=8$ mice/group). (D) Double immunofluorescence revealed the infiltration of TLR2-expressing macrophages in the lesions in the brachiocephalic arteries of $A p o E^{-/-}$mice aged 12 to 24 weeks that were placed on atherogenic diets from 6 weeks of age. The scale bars represent $20 \mu \mathrm{m}$. At the indicated ages, the $A p o E^{-/-} T / r 2^{-/-}$mice displayed significantly reduced $\mathrm{CHOP}(\mathrm{E}), \mathrm{Bcl}-2(\mathrm{~F})$, and cleaved caspase-3 (G) expression in the aortas. The data are shown as the mean \pm SEM ( $n=4-6$ mice/group). ${ }^{b} P<0.05$ vs the $A p o E^{-/-}$mice at the same age. ${ }^{e} P<0.05$ vs the $A p o E^{-/-}$mice aged 12 weeks on the chow diet.

opment of atherosclerosis. TLR2 has been demonstrated to participate in this process ${ }^{[11,27]}$. Consistent with previous studies of the early phases of atherosclerosis, blocking TLR2 activity reduced the infiltration of macrophages into plaques; 
the expression of inflammatory mediators, including MMP-2, TNF- $\alpha$ and IL-6; and the inactivation of the transcription factor NF-KB ${ }^{[20,21,23]}$. Moreover, TLR2 antagonism also inhibited the expression of the immunosuppressive mediators IL-10 and transcription factor Stat3, indicating that the benefits of blocking TLR2 are dependent on the extensive inhibition of inflammation. This study provides the first evidence for TLR2induced inflammation in advanced lesions, which would complement the previously revealed contributory role of TLR2 in early atherosclerosis.

TLR2 signaling activates pro-inflammatory genes in multiple cell types within the lesions, including endothelial cells, macrophages, vascular smooth muscle cells (VSMCs) and dendritic cells, but the key determinants of atherogenesis and plaque rupture are still unknown. Curtiss et al reported that TLR2 deficiency in BM-derived cells generated no impact on lesion size in $\mathrm{Ldlr}^{-/-}$mice during early atherosclerosis ${ }^{[18]}$. Consistent with this finding, the same group also demonstrated that the dramatic up-regulation of TLR2 is confined to endothelial cells in disturbed flow regions and does not occur in intimal leukocytes during the early progression of the disease ${ }^{[28]}$. These data suggest that endothelial TLR2 expression plays a key role in the initiation of atherosclerosis. However, TLR2 has been detected both in the endothelium and in inflammatory cells that infiltrated advanced atherosclerotic plaques obtained from patients undergoing endarterectomy ${ }^{[16]}$. Therefore, we hypothesized that the progression of advanced atherosclerosis involves the expression and activation of TLR2 in immune cells. Activated VSMC is another important contributor to atherogenesis and restenosis by multiple mechanisms, including extracellular matrix synthesis, release of pro-inflammatory mediators, proliferation and migration. Endogenous TLR2 ligands, such as heat shock protein 60 , could stimulate venous SMC proliferation through TLR2 and TLR $4^{[29]}$. In addition, ligand-activated TLR2 drives VSMC migration dependent on the production of IL- $6^{[30]}$. Using mouse models Schoneveld et al demonstrated that the vascular intimal hyperplasia, which is characterized by VSMC migration and proliferation, is enhanced by TLR2 activation ${ }^{[31]}$. However, VSMC is required for the structural integrity of the fibrous cap in advanced atherosclerosis to prevent the development of unstable lesions. Apoptosis of VSMC may also lead to plaque instability and rupture, which may result in acute atherothrombotic events ${ }^{[32]}$. The plaque content of VSMC is therefore considered an important determinant of plaque stability. In this study we found that the accumulation of VSMC was significantly increased in advanced plaques, thereby maintaining the plaque stability after blocking TLR2 activity. Moreover, the inhibition of VSMC proliferation and migration may also occur in advanced plaques because TLR2 blockade accompanies the decreased expression of IL-6 in aorta. It is unknown specifically how VSMC participates in the complex progress of advanced atherosclerosis and how TLR2 blockade affects it. An extensive study to focus on these issues should be taken in the near future.

Plaque instability is an important feature of advanced ath- erosclerosis. Although the mechanisms underlying plaque rupture remain elusive, many factors have been demonstrated to contribute to this process, including macrophage accumulation, high levels of pro-inflammatory mediators, excess activation of MMPs, and apoptosis of VSMCs and macrophage. In addition to the regulation of inflammatory responses, we observed that TLR2 blockade decreased macrophage apoptosis using TUNEL analysis and immunofluorescence.

Recently, ER stress-induced macrophage apoptosis has been considered to be a key event in plaque rupture ${ }^{[33,34]}$. Hypoxia-, oxidative stress-, and hypoglycemia-induced ER stress is universal in various diseases, including atherosclerosis. Sustained ER stress may result in apoptosis in a variety of cell types, including macrophages, through the activation of its downstream transcription factor CHOP. Generally, apoptotic cells are rapidly disposed via phagocytotic clearance by macrophages that have infiltrated into tissues. However, apoptotic macrophages result in a loss of phagocytic functions in advanced atherosclerotic lesions. Dying macrophages become necrotic, and cellular debris left in the plaques causes the necrotic core to expand. Many CHOP-expressing macrophages have been demonstrated to undergo apoptosis in advanced lesions in both humans and mice ${ }^{[35-37]}$. In addition to macrophage apoptosis, ER stress also enhances the expression of TLR 2 and the production of TLR-2 dependent pro-inflammatory cytokines in vitro and in vivo, suggesting a contributory role of ER stress in the regulation of TLR2dependent inflammation ${ }^{[38]}$. In contrast, TLR2 activation by atherogenic lipoproteins may promote the apoptosis of ERstressed macrophages and plaque necrosis during advanced atherosclerosis ${ }^{[15]}$. TLR2 activation acts in a synergistic fashion with ER stress to produce pro-inflammatory cytokines ${ }^{[39]}$. These data suggest that TLR2 signaling is associated with ER stress, although the exact mechanism remains unclear.

In our study, decreased $\mathrm{CHOP}$ and macrophage co-localization was observed in the lesions of the treated groups, suggesting that the plaque-stabilizing effect of TLR2 blockade may rely on the inhibition of ER stress-induced macrophage apoptosis. Given that there is no direct evidence for the impact of TLR2 signaling on ER stress in atherosclerosis, we provided

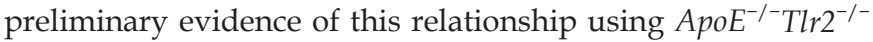
mice with atherosclerosis accelerated by $\mathrm{HC}$ diets. We confirmed that cell apoptosis and the expression of $\mathrm{CHOP}$ were significantly inhibited and that the expression of Bcl-2, a key cell survival molecule that is related to downstream CHOPmediated apoptosis, was elevated in $A p o E^{-/-} \mathrm{Tlr}^{-/-}$mice. Furthermore, we found that during the early phases of atherosclerosis in ApoE-deficient mice at 12 weeks of age, TLR2 expression was mainly detected in the endothelial and smooth muscle cells within the plaques, which was consistent with the results of other studies ${ }^{[28]}$. After another 6 to 12 weeks on the HC diet, the accumulation of vessel-infiltrating macrophages was dramatically increased, and the co-localization of TLR2 and macrophages was also detected in atherosclerotic vessels. Thus, TLR2 expressed on macrophages in advanced atherosclerotic lesions mediates cell apoptosis triggered by endog- 
enous stimuli, especially excess ER stress, and contributes to the growth of necrotic cores and plaque instability.

In summary, we have demonstrated that the knockout of TLR2 gene in $A p o E^{-/-}$mice reduced the plaque size and increased plaque stability in the brachiocephalic artery during advanced atherosclerosis as a result of the inhibition of inflammation and of ER-stress-induced macrophage apoptosis. Notably, the treatment of these mice with a TLR2-neutralizing antibody produced therapeutic beneficial effects and may serve as a promising therapeutic strategy against advanced atherosclerosis. Because the enhanced expression of TLR2 on endothelial cells is required for disease initiation, TLR2 may mediate or amplify the apoptotic signals induced by ER stress in advanced atherosclerotic lesions. Our work suggests that pharmacological blockade of TLR2 signaling is an attractive approach for the future treatment of atherosclerosis.

\section{Acknowledgements}

This study was supported by grants from the National Natural Science Foundation of China (81273529), Major Program of National Natural Science Foundation (81030056), International Corporation Project supported by the Ministry of Science and Technology (2010DFB32900), Program for Changjiang Scholars and Innovative Research Team in University (PCSIRT, № IRT1007), Creation of Major New Drugs (2009ZX09301-003-13) and Beijing Key Laboratory of New Drug Mechanisms and Pharmacological Evaluation Study (№ BZ0150).

\section{Author contribution}

Xiao-xing WANG and Zhuo-wei HU designed the study; Xiaoxing WANG, Xiao-xi LV, and Hui-min YAN contributed to the development of methodology; Xiao-xing WANG, Xiao-xi LV, Jia-ping WANG, Hui-min YAN, Zi-yan WANG, Han-zhi LIU, and Xiao-ming FU performed the experiments; Xiao-xing WANG and Zhuo-wei HU analyzed the data and wrote the paper.

\section{References}

1 Lopez AD, Mathers CD, Ezzati M, Jamison DT, Murray CJ. Global and regional burden of disease and risk factors, 2001: systematic analysis of population health data. Lancet 2006; 367: 1747-57.

2 Hansson GK, Robertson AK, Söderberg-Nauclér C. Inflammation and atherosclerosis. Ann Rev Pathol 2006; 1: 297-329.

3 Yan ZQ, Hansson GK. Innate immunity, macrophage activation, and atherosclerosis. Immunol Rev 2007; 219: 187-203.

4 Shah PK. Molecular mechanisms of plaque instability. Curr Opin Lipidol 2007; 18: 492-9.

5 Galkina E, Ley K. Immune and inflammatory mechanisms of atherosclerosis. Ann Rev Immunol 2009; 27: 165-97.

6 Bea F, Kreuzer J, Preusch M, Schaab S, Isermann B, Rosenfeld ME, et al. Melagatran reduces advanced atherosclerotic lesion size and may promote plaque stability in Apolipoprotein E-deficient mice. Arterioscler Thromb Vascul Biol 2006; 26: 2787-92.

7 Rosenfeld ME, Averill MM, Bennett BJ, Schwartz SM. Progression and disruption of advanced atherosclerotic plaques in murine models. Curr Drug Targets 2008; 9: 210-6.

8 Kawai T, Akira S. TLR signaling. Cell Death Differ 2006; 13: 816-25.
9 Kanzler H, Barrat FJ, Hessel EM, Coffman RL. Therapeutic targeting of innate immunity with Toll-like receptor agonists and antagonists. Nat Med 2007; 13: 552-9.

10 Curtiss LK, Tobias PS. The toll of toll-like receptors, especially toll-like receptor 2, on murine atherosclerosis. Curr Drug Targets 2007; 8: 1230-8.

11 Michelsen KS, Arditi M. Toll-like receptor signaling and atherosclerosis. Curr Opin Hematol 2006; 13: 163-8.

12 Tsan MF, Gao B. Endogenous ligands of Toll-like receptors. J Leukoc Biol 2004; 76: 514-9.

13 Tian J, Avalos AM, Mao SY, Chen B, Senthil K, Wu H, et al. Toll-like receptor 9-dependent activation by DNA-containing immune complexes is mediated by HMGB1 and RAGE. Nat Immunol 2007; 8: 487-96.

14 Akira S, Takeda K. Toll-like receptor signalling. Nat Rev Immunol 2004; 4: 499-511.

15 Seimon TA, Nadolski MJ, Liao X, Magallon J, Nguyen M, Feric NT, et al. Atherogenic lipids and lipoproteins trigger CD36-TLR2-dependent apoptosis in macrophages undergoing endoplasmic reticulum stress. Cell Metabolism 2010; 12: 467-82.

16 Edfeldt K, Swedenborg J, Hansson GK, Yan ZQ. Expression of toll-like receptors in human atherosclerotic lesions: a possible pathway for plaque activation. Circulation 2002; 105: 1158-61.

17 Monaco C, Gregan SM, Navin TJ, Foxwell BMJ, Davies AH, Feldmann M. Toll-like receptor-2 mediates inflammation and matrix degradation in human atherosclerosis. Circulation 2009; 120: 2462-9.

18 Mullick AE, Tobias PS, Curtiss LK. Modulation of atherosclerosis in mice by toll-like receptor 2. J Clin Invest 2005; 115: 3149-56.

19 Liu X, Ukai T, Yumoto H, Davey M, Goswami S, Gibson FC 3rd, et al. Toll-like receptor 2 plays a critical role in the progression of atherosclerosis that is independent of dietary lipids. Atherosclerosis 2008; 196: 146-54.

20 Yang HZ, Cui B, Liu HZ, Chen ZR, Yan HM, Hua F, et al. Targeting TLR2 attenuates pulmonary inflammation and fibrosis by reversion of suppressive immune microenvironment. J Immunol 2009; 182: 692-702.

21 Ma Y, Zhang X, Bao H, Mi S, Cai W, Yan H, et al. Toll-like receptor (TLR) 2 and TLR4 differentially regulate doxorubicin induced cardiomyopathy in mice. PLoS One 2012; 7: e40763.

22 Hayashi C, Madrigal AG, Liu X, Ukai T, Goswami S, Gudino CV, et al. Pathogen-mediated inflammatory atherosclerosis is mediated in part via Toll-like receptor 2-induced inflammatory responses. J Inn Immunity 2010; 2: 334-43.

23 Madan M, Amar S. Toll-like receptor-2 mediates diet and/or pathogen associated atherosclerosis: proteomic findings. PLoS One 2008; 3 : e3204.

24 Rosenfeld ME, Polinsky P, Virmani R, Kauser K, Rubanyi G, Schwartz $\mathrm{SM}$. Advanced atherosclerotic lesions in the innominate artery of the ApoE knockout mouse. Arterioscler Thromb Vasc Biol 2000; 20: 2587-92.

25 Zadelaar S, Kleemann R, Verschuren L, de Vries-Van der Weij J, van der Hoorn J, Princen HM, et al. Mouse models for atherosclerosis and pharmaceutical modifiers. Arterioscler Thromb Vasc Biol 2007; 27: 1706-21.

26 Preusch MR, Bea F, Yang SH, Kreuzer J, Isermann B, Pedal I, et al. Long-term administration of 3-deazaadenosine does not alter progression of advanced atherosclerotic lesions in apolipoprotein E-deficient mice. J Cardiovas Pharmacol 2007; 50: 206-12.

27 Björkbacka $\mathrm{H}$. Multiple roles of Toll-like receptor signaling in atherosclerosis. Curr Opin Lipidol 2006; 17: 527-33.

28 Mullick AE, Soldau K, Kiosses WB, Bell TA, Tobias PS, Curtiss LK. 
Increased endothelial expression of Toll-like receptor 2 at sites of disturbed blood flow exacerbates early atherogenic events. J Exp Med 2008; 205: 373-83.

29 de Graaf R, Kloppenburg G, Kitslaar PJ, Bruggeman CA, Stassen F. Human heat shock protein 60 stimulates vascular smooth muscle cell proliferation through Toll-like receptors 2 and 4 . Microbes Infect 2006; 8: 1859-65.

30 Lee G, Chang Y, Wu J, Wu M, Wu K, Yet S, et al. TLR 2 induces vascular smooth muscle cell migration through cAMP response element-binding protein-mediated interleukin- 6 production. Arterioscler Thromb Vasc Biol 2012; 32: 2751-60.

31 Schoneveld AH, Oude Nijhuis MM, van Middelaar B, Laman JD, de Kleijn DP, Pasterkamp G. Toll-like receptor 2 stimulation induces intimal hyperplasia and atherosclerotic lesion development. Cardiovasc Res 2005; 66: 162-9.

32 Clarke MC, Figg N, Maguire JJ, Davenport AP, Goddard M, Littlewood TD, et al. Apoptosis of vascular smooth muscle cells induces features of plaque vulnerability in atherosclerosis. Nat Med 2006; 12 : 1075-80.

33 Myoishi M, Hao H, Minamino T, Watanabe K, Nishihira K, Hatakeyama $\mathrm{K}$, et al. Increased endoplasmic reticulum stress in atherosclerotic plaques associated with acute coronary syndrome. Circulation 2007;
116: 1226-33.

34 Tabas I, Ron D. Integrating the mechanisms of apoptosis induced by endoplasmic reticulum stress. Nat Cell Biol 2011; 13: 184-90.

35 Tsukano H, Gotoh T, Endo M, Miyata K, Tazume H, Kadomatsu T, et al. The endoplasmic reticulum stress-C/EBP Homologous protein pathway-mediated apoptosis in macrophages contributes to the instability of atherosclerotic plaques. Arterioscler Thromb Vasc Biol 2010; 30: 1925-32.

36 Tabas I. Macrophage apoptosis in atherosclerosis: consequences on plaque progression and the role of endoplasmic reticulum stress. Antioxidants Redox Signaling 2009; 11: 2333-9.

37 Thorp E, Li G, Seimon TA, Kuriakose G, Ron D, Tabas I. Reduced apoptosis and plaque necrosis in advanced atherosclerotic lesions of $\mathrm{ApoE}^{-/-}$and $\mathrm{Ldll}^{-/-}$mice lacking CHOP. Cell Metabolism 2009; 9: 474-81.

38 Shogo S, Tomoaki K, Tsuyoshi S, Mary AS, Takashi S, Kenji W, et al. Endoplasmic reticulum stress increases the expression and function of toll-like receptor- 2 in epithelial cells. Biochem Biophys Res Commun 2010; 402: 235-40.

39 Martinon F, Chen X, Lee AH, Glimcher LH. TLR activation of the transcription factor XBP1 regulates innate immune responses in macrophages. Nat Immunol 2010; 11: 411-8. 